astro-ph/9811181

FTUV/98-82, IFIC/98-83

\title{
Type-II Supernovae and Neutrino Magnetic Moments
}

\author{
H. Nunokawa ${ }^{1 *}$, R. Tomàs ${ }^{2 \dagger}$ and J. W. F. Valle ${ }^{2 \ddagger}$ \\ ${ }^{1}$ Instituto de Física Gleb Wataghin \\ Universidade Estadual de Campinas - UNICAMP \\ 13083-970 Campinas SP Brasil \\ ${ }^{2}$ Instituto de Física Corpuscular - C.S.I.C. \\ Departament de Física Teòrica, Universitat de València \\ 46100 Burjassot, València, SPAIN \\ URL http://neutrinos.uv.es
}

(November, 1998)

\begin{abstract}
The present solar and atmospheric neutrino data together with the LSND results and the presence of hot dark matter (HDM) suggest the existence of a sterile neutrino at the eV scale. We have reanalysed the effect of resonant sterile neutrino conversions induced by neutrino magnetic moments in a type-II supernova. We analyse the implications of $\nu_{e}-\nu_{s}$ and $\bar{\nu}_{e}-\bar{\nu}_{s}\left(\nu_{s}\right.$ denotes sterile neutrino) conversions for the supernova shock re-heating, the detected $\bar{\nu}_{e}$ signal from SN1987A and the $r$-process nucleosynthesis hypothesis. Using reasonable magnetic field profiles we determine the sensitivity of these three arguments to the relevant neutrino parameters, i.e. the value of the transition magnetic moment and the $\nu_{e}-\nu_{s}$ mass difference $\Delta m^{2} \equiv \Delta m_{L S N D / H D M}^{2}$

PACS numbers: 14.60.Pq, 14.60.St, $13.15+\mathrm{g}$
\end{abstract}

\section{INTRODUCTION}

It was recognized a while ago [1] that the simplest model to reconcile the existence of a hot dark matter (HDM) component in the Universe [2] and possibly incorporating an explanation of the LSND results [3] with the solar [4] and atmospheric neutrino data [5] is to invoke a fourth sterile (electroweak singlet) neutrino at the eV scale. Recently there have been many papers on this scheme and its variants as well as phenomenology [6]. The effects of active to sterile neutrino transitions induced by the mixing on supernova physics have been studied in a number of papers [7],8].

Here we also consider the relevance of such active to sterile neutrino conversion $\nu_{e} \rightarrow \nu_{s}$ ( $\nu_{s}$ denote sterile neutrino) but induced by a nonzero transition magnetic moment (TMM), similar to the transition magnetic moment between two active neutrino flavours [9]. Such TMM leads to a neutrino spin-flavour precession (SFP) effect similar to the active-active case [9]. The same way as the SFP effect may take place resonantly in the presence of matter

\footnotetext{
*E-mail: nunokawa@ifi.unicamp.br

${ }^{\dagger}$ E-mail: ricard@flamenco.ific.uv.es

${ }^{\ddagger}$ E-mail: valle@flamenco.ific.uv.es
} 
[10], like MSW effect [11], so does the active-sterile SFP. Here we adopt the general case where $\nu_{e}$ and $\nu_{s}$ are two independent Majorana particles. However, the sterile state can be a right-handed component of a Dirac neutrino. In this case, $\nu_{s}\left(\bar{\nu}_{s}\right)$ should be regarded as a particle carrying lepton number -1 (1) in our notation.

Although expected to be small, e.g. if the neutrino mass is introduced a la Dirac in the same way as the charged fermion masses in the standard electroweak theory the resulting neutrino magnetic moment is known to be very small [12], $\mu_{\nu} \sim 3 \times 10^{-19}\left(m_{\nu} / 1 \mathrm{eV}\right) \mu_{B}$ where $m_{\nu}$ is the neutrino mass and $\mu_{B}$ denotes the Bohr magneton. However, several attempts have been made to construct various mechanisms to induce a large neutrino magnetic moment of order $\sim 10^{-11} \mu_{B}[13]$. In fact this is a natural possibility in the context of radiative models of neutrino mass, such as that of the sterile neutrino models of refs. [1, 14, 15].

Let us first recall here the previous bounds on the neutrino magnetic moments from laboratory experiments as well as from astrophysical considerations. Starting with laboratory limits, the upper bound on the neutrino magnetic moments comes from the $\bar{\nu}_{e} e$ scattering experiments which give 16],

$$
\mu_{\nu}<1.8 \times 10^{-10} \mu_{B}
$$

This bound applies to the direct or transition magnetic moment of Dirac neutrinos, as well as to the transition magnetic moment of Majorana neutrinos.

From the SN1987A neutrino observations the bounds on dipole magnetic moment of Dirac neutrinos has been derived by considering the helicity-flipping scattering processes such as $\nu_{L} e^{-} \rightarrow \nu_{R} e^{-}$and $\nu_{L} p \rightarrow \nu_{R} p$ inside the supernova core 17]. By requiring that the $\nu_{R}$ luminosity should be not too large in order to account for the observed neutrino data from SN1987A in Kamiokande II [19] and IMB [20] detectors, the constraint $\mu_{\nu} \lesssim 10^{-12} \mu_{B}$ is obtained. Similar upper bound has been obtained also in a recent paper [18] by considering neutrino helicity flipping process through photon Landau damping in a dense relativistic plasma in a supernova core. It has also been discussed [17] that this bound could be improved to $\mu_{\nu} \lesssim 10^{-13} \mu_{B}$ by taking into account the absence of higher energy neutrino events which would be expected due to the spin rotation of higher energy $\nu_{R}$ produced in the core to $\nu_{L}$ in the galactic magnetic field. Let us note that these discussion also applies to the transition magnetic moment of Dirac neutrinos as well as to the ones which connects active and sterile Majorana neutrinos if one can neglect the mass squared difference $\Delta m^{2}$ in the process discussed above. However, it has been pointed out that 21 these bounds could be invalid if the resonant re-conversion of neutrinos take place in the supernova.

One could also obtain limits from the arguments of excessive cooling of red giant stars due to the neutrino emission induced by the neutrino magnetic moment. Ref. [22] gives the bound,

$$
\mu_{\nu}<3 \times 10^{-12} \mu_{B}
$$

which also applies both to Dirac magnetic moments, as well as for Majorana transition moments.

In this paper we show that a non-zero $\nu_{e}-\nu_{s}$ transition magnetic moment (TMM) would have an important effects in the presence of the strong magnetic fields $10^{15}$ Gauss during a SN explosion 1 since there can be a resonant spin-flavour precession (RSFP) between the active and the sterile neutrino flavour, similar to what occurs for the active-active case. The latter has been extensively considered in the literature [25]. We show how $\nu_{e}-\nu_{s}$ resonant conversions induced by the active-sterile TMM enhance the $r$-process nucleosynthesis in all of the mass range relevant for the neutrino hot dark matter scenario [2]. On the other hand we estimate the restrictions on neutrino active-sterile

\footnotetext{
${ }^{1}$ It would also have implications for the solar neutrino problem 23.24.
} 
TMM from SN1987A data, and investigate the influence of these $\nu_{e}-\nu_{s}$ TMM-induced conversions in the shock revival problem. For previous related work see refs. 17,21,26,27]. In particular, ref. [27] has considered $\nu_{e}-\nu_{s}$ TMM-induced RSFP in the context of SN physics for the case of random magnetic fields, in which case the conversion occurs in a non-periodic regime. Here we are considering the case of regular fields, and we discuss novel issues related to SN physics which were not considered in [27]. Throughout this paper we assume $\mu_{\nu}<10^{-12} \mu_{B}$ so that the neutrino helicity flipping process in the core 17, 18], discussed above can be neglected (see the discussion in Sec. IV for the case if $\left.\mu_{\nu}>10^{-12} \mu_{B}\right)$.

In Sec. II we briefly describe the picture of the neutrino propagation in matter and the resonant $\nu_{e}-\nu_{s}$ conversion [11]. Sec. III.A discusses the implications of $\nu_{e}-\nu_{s}$ conversions for the neutrino re-heating mechanism. In Sec. III.B we analyse the impact of our scenario in the later epoch of supernova evolution (few seconds after the core bounce) for SN (anti-) neutrino detection rates (Sec. III.B), as well as for $r$-process nucleosynthesis (Sec. III.C). In Sec. IV we summarize our results.

\section{THE ACTIVE-STERILE NEUTRINO RESONANT SPIN PRECESSION}

In our discussion we only consider the $\nu_{e} \rightarrow \nu_{s}$ and $\bar{\nu}_{e} \rightarrow \bar{\nu}_{s}$ conversion channels, where $\nu_{s}\left(\bar{\nu}_{s}\right)$ is a sterile neutrino, due to resonant spin precession. For simplicity we neglect in what follows the mixing between $\nu_{e}$ and $\nu_{s}$ so that the $4 \times 4$ evolution Hamiltonian for the neutrino system [9] reduces to an effective $2 \times 2$ system. Moreover we consider the $\Delta m^{2}=m_{s}^{2}-m_{e}^{2}>0$ case, i.e., sterile state is heavier. The evolution of the $\nu_{e}-\nu_{s}$ system in the matter background with non-zero magnetic field is determined by the following Schröedinger-like equation,

$$
i \frac{\mathrm{d}}{\mathrm{d} r}\left(\begin{array}{c}
\nu_{e} \\
\nu_{s}
\end{array}\right)=\left(\begin{array}{cc}
V_{e}-\frac{\Delta m^{2}}{2 E_{\nu}} & \mu_{\nu} B \\
\mu_{\nu} B & 0
\end{array}\right)\left(\begin{array}{c}
\nu_{e} \\
\nu_{s}
\end{array}\right)
$$

where $\mu_{\nu}$ is the neutrino magnetic moment and $B$ is the magnetic field strength perpendicular to the neutrino trajectory. The effective potential $V_{e}$ for $\nu_{e}$ arises from the coherent forward neutrino scattering off-matter constituents [11] and is given by ?

$$
\begin{aligned}
& V_{e}=\frac{\sqrt{2} G_{F} \rho}{m_{N}}\left(Y_{e}-\frac{1}{2} Y_{n}\right)=\frac{\sqrt{2} G_{F} \rho}{2 m_{N}}\left(3 Y_{e}-1\right), \\
& Y_{e} \equiv \frac{n_{e}}{n_{e}+n_{n}}, \quad Y_{n}=1-Y_{e} .
\end{aligned}
$$

Note that there is no potential for $\nu_{s}$, i.e., $V_{s}=0$. Here $G_{F}$ is the Fermi constant, $\rho$ is the matter density, $m_{N}$ is the nucleon mass and $n_{e}$ and $n_{n}$ are the net electron and the neutron number densities in matter, respectively. Note that charge neutrality $n_{p}=n_{e}$ is assumed. For the $\bar{\nu}_{e} \rightarrow \bar{\nu}_{s}$ system the matter potential just change its sign.

The resonance condition is:

$$
V_{e}=\frac{\Delta m^{2}}{2 E_{\nu}}
$$

Let us note that for $\Delta m^{2}>0$, either $\nu_{e} \rightarrow \nu_{s}$ (for $V_{e}>0$ i.e. $Y_{e}>1 / 3$ ) or $\bar{\nu}_{e} \rightarrow \bar{\nu}_{s}$ (for $V_{e}<0$ i.e. $Y_{e}<1 / 3$ ) conversions take place. This is important because, as we discussed in ref. [8], in the region above the neutrinosphere the matter potential $V_{e}$ changes its sign due to the different chemical content.

\footnotetext{
${ }^{2}$ It is reasonable to neglect the contributions from the neutrino background to the effective potential, since the neutrino densities in the relevant regions are small.
} 


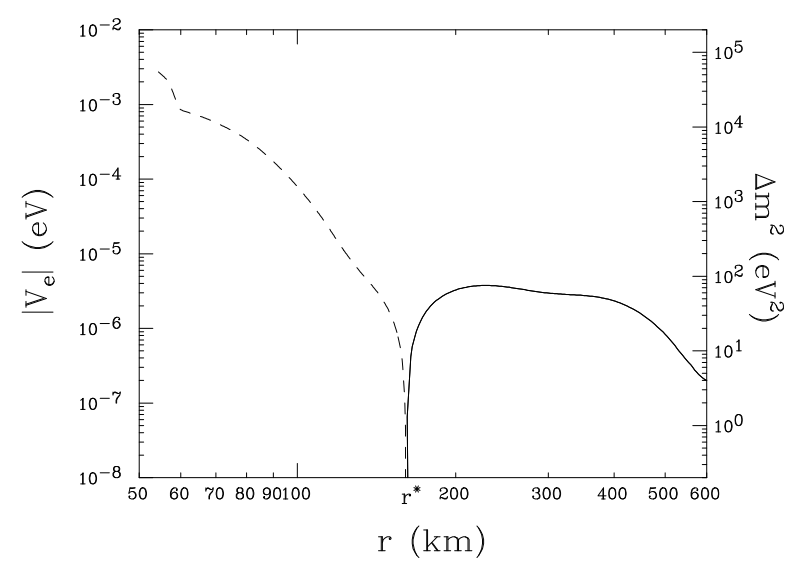

FIG. 1. Typical $\left|V_{e}\right|$ profile versus the radial distance $r$ at $t<1 \mathrm{~s}$ after the core bounce. The solid and dashed lines correspond to positive and negative potential, respectively, and the position where $V_{e}=0$ is denoted by $r^{*}$. The $\Delta m^{2}$ values for which a $E=10 \mathrm{MeV}$ neutrino undergoes resonant conversion, for the $\left|V_{e}\right|$ value on the left ordinate is indicated in the right ordinate. This is relevant for the shock revival considerations.

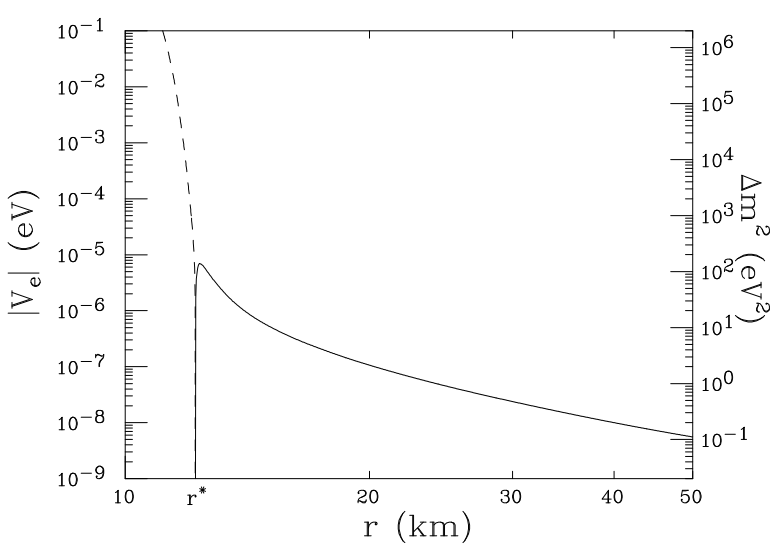

FIG. 2. Same as Fig. 1 for $t>1 \mathrm{~s}$. This is relevant for the $r$-process and $\bar{\nu}_{e}$ signal considerations.

In Figs. 1 (earlier epoch) and 2 (later epoch) we reproduce the typical profiles of corresponding $\left|V_{e}\right|$ in the region of interest, taken from ref. [8]. They are obtained from the $Y_{e}$ and $\rho$ profiles taken from Wilson's supernova model outside the neutrinosphere, at two different times, $t<1 \mathrm{~s}$ and $t>1 \mathrm{~s}$ post-bounce. There is a point where $Y_{e}$ takes the value $1 / 3$ (i.e. $V_{e}=0$ ), as indicated by $r^{*}$. This position corresponds to $r^{*} \approx 160 \mathrm{~km}$ and $12 \mathrm{~km}$ for the earlier and later epochs, respectively. Clearly, the effective potential $V_{e}$ changes its sign from negative to positive at the point $r^{*}$.

The resonance condition in eq. (5) provides the $\Delta m^{2}$ value for which neutrinos with some given energy can experience the resonance for a certain value of the potential (or equivalently, at some position $r$ ). For convenience, in the right ordinate of the Figs. 1 and 2 we have also indicated the corresponding $\Delta m^{2}$ values for which neutrinos under go resonance, for typical neutrino energy $E=10 \mathrm{MeV}$. We see that for $\Delta m^{2} \gtrsim 10^{2} \mathrm{eV}^{2}$ only $\bar{\nu}_{e} \rightarrow \bar{\nu}_{s}$ conversions can occur, and this happens in the region where $Y_{e} \leq 1 / 3$. On the other hand for $\Delta m^{2} \lesssim 10^{2} \mathrm{eV}^{2}$ three resonances may occur. The $\bar{\nu}_{e}$ 's are first converted, say at $r_{1}<r^{*}$, then there are two resonance points at $r_{2}\left(\approx r_{1}\right)$ and $r_{3}$, where $r_{3}>r_{2}>r^{*}$ (i.e. in the region where $Y_{e}>1 / 3$ ), for the $\nu_{e} \rightarrow \nu_{s}$ channel. A schematic level crossing diagram for $\nu_{e}-\nu_{s}$ and $\bar{\nu}_{e}-\bar{\nu}_{s}$ system may be seen explicitly in Fig. 3 of ref. [8]. Note that the $\nu_{s}$ 's originated from the first $\nu_{e}$ 
conversion $\left(\right.$ at $\left.r_{2}\right)$ can be re-converted into $\nu_{e}$ 's at the second resonance (at $\left.r_{3}\right)$.

In what follows we will employ the simple Landau-Zener approximation [28,29] in order to estimate the survival probability after the neutrinos cross the resonance. Under this approximation, the $\nu_{e}\left(\right.$ or $\left.\bar{\nu}_{e}\right)$ survival probability is given by,

$$
\begin{aligned}
P & =\exp \left(-\frac{\pi^{2}}{2} \frac{\Delta r}{L_{m}^{\mathrm{res}}}\right) \\
& \approx \exp \left[-1.1 \times 10^{-6}\left(\frac{\mu B}{\mu_{B} \mathrm{G}}\right)_{r e s}^{2}\left(\frac{\mathrm{d} V_{e}}{\mathrm{~d} r} \times \frac{\mathrm{km}}{\mathrm{eV}}\right)_{r e s}^{-1}\right]
\end{aligned}
$$

where $L_{m}^{\text {res }}$ is the neutrino oscillation length at resonance. Notice that for $\Delta r / L_{m}^{\text {res }}>1$ the resonant neutrino conversion will be adiabatic $[1]$.

As we discussed in ref. [8], depending on the $\Delta m^{2}$ values, the $\nu_{e}-\nu_{s}$ system may encounter two resonances. In order to take this into account we compute the $\nu_{e}$ survival probability after the second resonance as follows,

$$
P\left(\nu_{e} \rightarrow \nu_{e}\right)=P\left(r_{2}\right) P\left(r_{3}\right)+\left[1-P\left(r_{2}\right)\right]\left[1-P\left(r_{3}\right)\right]
$$

where $P\left(r_{2}\right)$ and $P\left(r_{3}\right)$ are the survival probabilities calculated according to the eq. (6) at the first and second resonance positions $r_{2}$ and $r_{3}$, respectively.

In this work we will neglect the spin precession due to the galactic magnetic field discussed in ref. [17], since for the relevant parameters of interest to us here the condition $\mu_{\nu} B_{G} \ll \Delta m^{2} / E$, where $B_{G} \sim 10^{-6}$ G is the galactic magnetic field, is always satisfied so that the spin precession in the galactic field is strongly suppressed.

\section{IMPLICATIONS FOR SUPERNOVA PHYSICS}

Following ref. [8] we now consider the possible impact of active-sterile neutrino conversions on different aspects of supernova astrophysics. We analyse processes taking place at the early epochs as well as at the cooling stage, in order to get a feeling for their sensitivity to the underlying neutrino mass-square difference, magnetic moments and magnetic fields. In the following we consider only epochs after the core bounce and the neutrino evolution in regions outside the neutrino-spheres, where resonant conversions take place.

\section{A. Shock Revival}

Here we are considering the earlier epoch $t<1$ second after core bounce. In the delayed explosion scenario 30,31 the neutrino energy deposition, occurring between the neutrino sphere and the site where the shock is stalled (about $500 \mathrm{~km}$ or so from the core) can re-start the shock and power the explosion. The disappearance of either $\nu_{e}$ or $\bar{\nu}_{e}$ due to the resonant spin precession into sterile states would reduce the energy deposition rate. The neutrino energy deposition rate $R$ at the stalled shock is defined as follows,

$$
\begin{aligned}
R & =\frac{Y_{n}^{\prime} \dot{E}_{\nu_{e} n}^{\prime}(t)+Y_{p}^{\prime} \dot{E}_{\bar{\nu}_{e} p}^{\prime}(t)}{Y_{n} \dot{E}_{\nu_{e} n}(t)+Y_{p} \dot{E}_{\bar{\nu}_{e} p}(t)}, \\
\dot{E}_{\nu N}(t) & \sim \int E_{\nu} \sigma_{\nu N} \phi^{0}\left(E_{\nu}\right) \mathrm{d} E_{\nu}, \quad \dot{E}_{\nu N}^{\prime}(t) \sim \int E_{\nu} \sigma_{\nu N} P\left(E_{\nu}\right) \phi^{0}\left(E_{\nu}\right) \mathrm{d} E_{\nu} .
\end{aligned}
$$


where the primed quantities $Y_{p}^{\prime}$ and $Y_{n}^{\prime}$ stand for the proton and neutron abundances calculated in the presence of active-sterile neutrino conversions. The un-primed ones correspond to the case where no conversion occurs. As an approximation, we have assumed, in eq. (9) that the neutrino energy spectra $\phi^{0}\left(E_{\nu}\right)$ are Fermi-Dirac with zero chemical potential but with different characteristic temperatures $T_{\nu}$ which depend on the neutrino flavour. As in our previous analysis [8] we choose $T_{\nu}$ for $\nu_{e}$ and $\bar{\nu}_{e}$ such that the typically predicted average energies, $\left\langle E_{\nu_{e}}\right\rangle=11 \mathrm{MeV}$ and $\left\langle E_{\bar{\nu}_{e}}\right\rangle=16 \mathrm{MeV}$ are obtained in the absence of any conversion. We assume a magnetic field profile $B(r)=B_{0}\left(r_{0} / r\right)^{n}$ where $B_{0}=10^{16} \mathrm{G}, n=2, r_{0}=100 \mathrm{~km}$ and $r$ is the distance from the center of the star. We have also studied the case where $n=3$ and obtained the similar results.

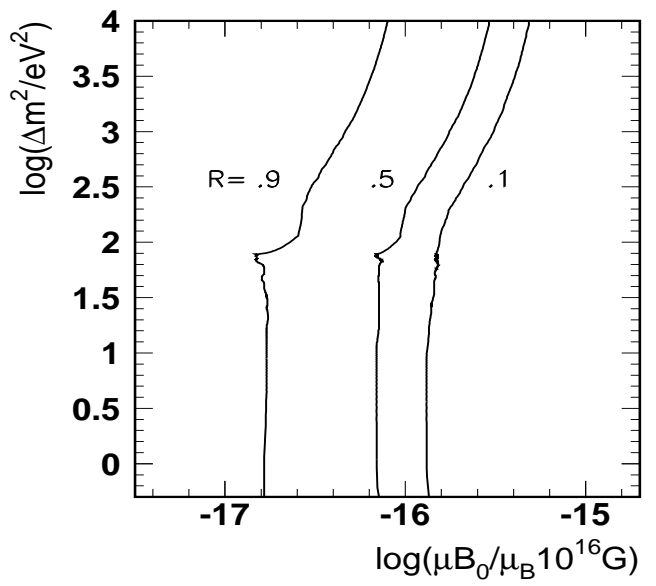

FIG. 3. Contour plot of the reheating rate $R$ in the $\Delta m^{2}-\mu B_{0}$ plane. The rate is normalized to 1 in the absence of any kind of neutrino conversion. Here $\mu_{B}$ denotes the Bohr magneton.

In Fig. 3 we plot the iso-contour of $R$ in terms of $\left(\Delta m^{2}, \mu B_{0}\right)$. We show the values of $\mu_{\nu} B_{0}$ in units of $\mu_{B} \cdot 10^{16}$ Gauss, where $10^{16} \mathrm{G}$ (at $r=100 \mathrm{~km}$ ) might be the maximally conceivable value [32]. This is taken as a reference value in order to illustrate the maximal sensitivity to the neutrino magnetic moment (though such a value of magnetic field may affect the supernova dynamics and self-consistent calculations would be required for its justification [33]). Thus we can say that if the neutrino re-heating is essential for successful supernova explosion the parameter region right to the curve, say $R=0.5$, is disfavoured.

\section{B. Implications for the detection of SN1987A $\bar{\nu}_{e}$ signal}

We are now in the later epoch $t>1$ second after core bounce, in the so-called Kelvin-Helmholtz cooling phase. The observed $\bar{\nu}_{e}$ events from the supernova SN1987A in Kamiokande II [19] and IMB [20] detectors, 11 and 8 events, respectively, are in good agreement with the theoretical expectations. Therefore, any significant conversion of $\bar{\nu}_{e}$ 's into a sterile neutrino species would be in conflict with this evidence. We consider the effect of active-sterile neutrino conversions both on the $\bar{\nu}_{e}$ signal in order to analyse the possible restrictions on neutrino parameters, the neutrino magnetic moment and the mass-squared difference.

We plot in Fig. $⿴$ three contours of the $\bar{\nu}_{e}$ survival probability $\langle P\rangle$, which is averaged over the Fermi-Dirac energy distribution, for the $\bar{\nu}_{e} \rightarrow \bar{\nu}_{s}$ conversion, in the $\left(\Delta m^{2}, \mu B_{0}\right)$ plane. In the plot the left, the middle and the right lines correspond to $\langle P\rangle=0.9,0.5$ and 0.1 , respectively. If we assume that the successful observation of the SN1987A signal implies that at least $50 \%$ of the expected $\bar{\nu}_{e}$ signal has been detected, one can conclude that all the portion right to the curve $P=0.5$ is ruled out. We note that due to the fact that the potential is much steeper in the 
later epoch than in the earlier epoch, the bound we obtained in Fig. 4 is much weaker than the sensitivity we have displayed in Fig. 3.

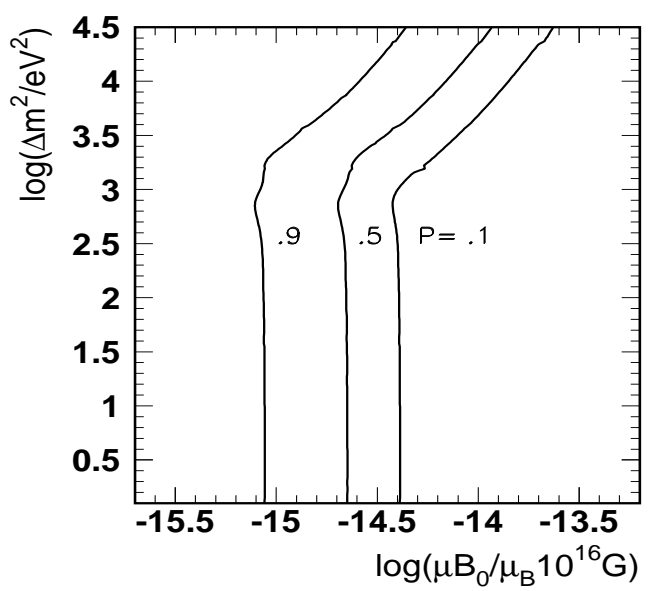

FIG. 4. Contour plots of the survival probability $P$ (figures at the curve) for the $\bar{\nu}_{e} \rightarrow \bar{\nu}_{s}$ conversion at $t>1 \mathrm{~s}$ after the bounce. The region to the right of the curve corresponding to $P=0.5$ can be excluded by the observation of the SN1987A $\bar{\nu}_{e}$ signal. We have assumed the magnetic profile to be $B(r)=B_{0}\left(r_{0} / r\right)^{n}$ where $r_{0}=10 \mathrm{~km}$ and $n=2$.

\section{Implications for $r$-process Nucleosynthesis}

Here, we consider the implications of resonant neutrino conversions for the supernova nucleosynthesis of heavy elements [34]8]. It has been proposed that the supernova could be the most promising site for heavy elements (mass number $A>70$ ) nucleosynthesis, the so-called $r$-process [35], in which neutrinos play very important rôle [36]. A necessary condition required for the $r$-process is $Y_{e}<0.5$ in the nucleosynthesis region. The $Y_{e}$ value in the $r$-process site is mainly determined by the following neutrino absorption reactions,

$$
\begin{aligned}
& \nu_{e}+n \rightarrow p+e^{e} \\
& \bar{\nu}_{e}+p \rightarrow n+e^{+} .
\end{aligned}
$$

Therefore, in the nucleosynthesis region we can write $Y_{e}$ as follows [34,

$$
Y_{e} \approx \frac{1}{1+P_{\bar{\nu}_{e}}\left\langle E_{\bar{\nu}_{e}}\right\rangle / P_{\nu_{e}}\left\langle E_{\nu_{e}}\right\rangle},
$$

From this expression, we notice that the $\bar{\nu}_{e} \rightarrow \bar{\nu}_{s}$ conversion leads to an increase of $Y_{e}$, whereas the $\nu_{e} \rightarrow \nu_{s}$ conversion acts in the opposite way. As we described in detail in ref. [8], depending on the $\Delta m^{2}$ range, one channel dominates over the other one, and $Y_{e}$ can be increased or decreased. Using again the Fermi-Dirac energy distribution to average the neutrino absorption rates, we have calculated the electron abundance $Y_{e}$ at the site where heavy elements nucleosynthesis is expected to take place as functions of $\left(\Delta m^{2}, \mu B_{0}\right)$.

We present our result in Fig. 5. For a successful $r$-process, the region above $Y_{e}>0.5$ is ruled out. On the other hand we find that the supernova nucleosynthesis could be enhanced in the region enclosed by the contour $Y_{e}=0.4$, similar to the results obtained in ref. [8]. This region is delimited by $\Delta m^{2} \lesssim 10^{2} \mathrm{eV}^{2}$ and $10^{-16} \lesssim \mu_{\nu} \lesssim 10^{-15}$ in units of $\mu_{B}$ for $B_{0}=10^{16} \mathrm{G}$. Inside the contour of $Y_{e}=0.33, Y_{e}$ might get stabilised to $1 / 3$ due to some feedback effect which we discussed in detail in ref. [B]. We see from the plot that the most promising mass range for the neutrino hot 
dark matter scenario [2], $\Delta m^{2} \lesssim 10 \mathrm{eV}^{2}$, lies inside that where the $r$-process nucleosynthesis is enhanced. Moreover, it is neither in conflict with the re-heating process (see Fig. 3), for $\mu_{\nu} B_{0} \lesssim\left(10^{-15} \mu_{B}\right) \cdot\left(10^{16} \mathrm{G}\right.$ ), nor with SN1987A observations (see Fig. 4).

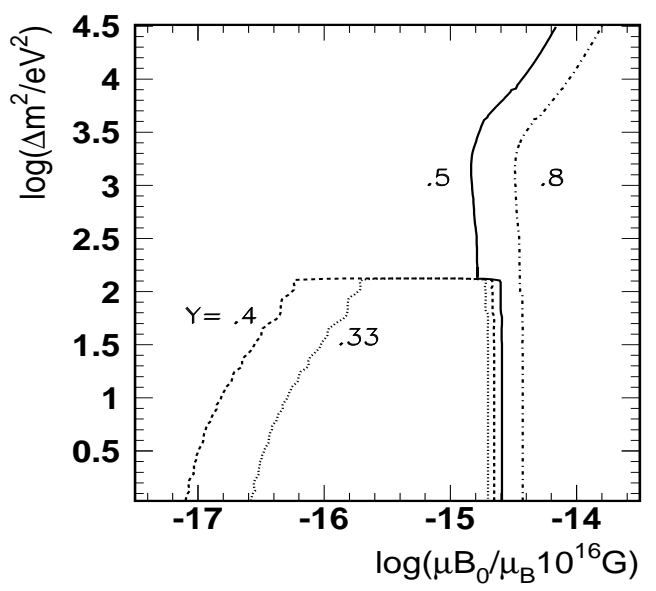

FIG. 5. Contour plots for the electron fraction $Y_{e}$ (figures at the curves) taking into account $\bar{\nu}_{e} \rightarrow \bar{\nu}_{s}$ and $\nu_{e} \rightarrow \nu_{s}$ conversions at $t>1 \mathrm{~s}$ after the bounce. The region to the right of the solid line labelled 0.5 is ruled out by the condition $Y_{e}<0.5$ necessary for $r$-process nucleosynthesis to occur. For the parameter region inside the $Y_{e}=0.4$ dotted contour $r$-process nucleosynthesis can be enhanced.

\section{CONCLUSIONS AND DISCUSSIONS}

In this paper we have considered the effect on supernova physics of having RSFP conversions induced by non-zero active-sterile TMM. We have investigated the sensitivity of the shock revival and $r$-process arguments, as well as estimated the restrictions from the observed SN1987A $\bar{\nu}_{e}$ detection rates. We have analysed the effect of RSFP conversions involving $\nu_{e}$ to $\nu_{s}$ or $\bar{\nu}_{e}$ to $\bar{\nu}_{s}$ in the region above the hot proto-neutron star in type II supernovae, assuming the simple magnetic field profile, $B(r)=B_{0}\left(r_{0} / r\right)^{n}(n=2,3)$ where $r_{0}$ is taken to be 100 and $10 \mathrm{~km}$ for earlier and later epoch, respectively. If the mass of the $\nu_{s}$ is in the cosmologically interesting range and the product of the TMM by the magnetic field just above the neutrinosphere, $\mu_{\nu} B_{0}$, is larger than $\sim\left(10^{-15} \mu_{B}\right) \times\left(10^{16} \mathrm{G}\right)$, then a significant fraction of $\nu_{e}$ and $\bar{\nu}_{e}$ would be converted into $\nu_{s}$ and $\bar{\nu}_{s}$, respectively, in the region outside neutrinosphere. Such conversion could lead to the depletion of $\nu_{e}$ and $\bar{\nu}_{e}$ fluxes, resulting in a suppression of the expected $\bar{\nu}_{e}$ signal in underground terrestrial detectors, in contradiction with the successful observation of the SN1987A $\bar{\nu}_{e}$ signal in the IMB and Kamiokande detectors. Hence, on this basis we can constrain the neutrino mass and neutrino magnetic moment by requiring that the total $\bar{\nu}_{e}$ flux during the thermal neutrino emission epoch should not be significantly depleted by $\bar{\nu}_{e} \rightarrow \bar{\nu}_{s}$ conversion, and can rule out the range $\mu_{\nu} B_{0} \gtrsim\left(10^{-15} \mu_{B}\right) \times\left(10^{16} \mathrm{G}\right)$.

The TMM-induced $\nu_{e}-\nu_{s}$ RSFP would also suppress the neutrino re-heating behind the stalled shock. We have found that for $\mu_{\nu} B_{0} \gtrsim\left(10^{-16} \mu_{B}\right) \times\left(10^{16} \mathrm{G}\right)$, the energy deposition by $\nu_{e}$ and $\bar{\nu}_{e}$ absorption reactions during the shock re-heating epoch $(t<1 \mathrm{~s}$ after the bounce) could be significantly decreased. From the $r$-process argument, for the parameter range $\Delta m^{2} \gtrsim 100 \mathrm{eV}^{2}$, where the $\bar{\nu}_{e} \rightarrow \overline{\nu_{s}}$ conversion is dominant, $Y_{e}$ at the nucleosynthesis site could become larger than 0.5 and hence $r$-process would be forbidden, leading to a disfavoured range, $\mu_{\nu} B_{0} \gtrsim\left(10^{-15} \mu_{B}\right) \times$ $\left(10^{16} \mathrm{G}\right)$. On the other hand, for $\Delta m^{2} \lesssim 100 \mathrm{eV}^{2}, r$-process nucleosynthesis could be enhanced due to the decrease of $Y_{e}$ down to the minimum value $1 / 3$ due to the efficient $\nu_{e} \rightarrow \nu_{s}$ conversion if the parameters are in the region $\left(10^{-16} \mu_{B}\right) \times\left(10^{16} \mathrm{G}\right) \lesssim \mu_{\nu} B_{0} \lesssim\left(10^{-15} \mu_{B}\right) \times\left(10^{16} \mathrm{G}\right)$ 
Throughout the above discussion we have neglected, for simplicity, the production of the sterile state due to the neutrino helicity-flipping processes in the core, which was discussed in refs. [17, 18]. This requires $\mu_{\nu} \lesssim 10^{-12} \mu_{B}$. Let us finally discuss briefly the case $\mu_{\nu}$ is larger than $10^{-12} \mu_{B}$ (but smaller than the laboratory limit (1)). As discussed in refs. 17.18], in this case the sterile states can be copiously produced by the helicity-flipping scattering processes in the SN core and they can escape freely to the outer region with higher energy.

For the earlier epoch, relevant for shock reheating, if the parameters $\left(\Delta m^{2}, \mu B_{0}\right)$ are in the region where the RSFP is efficient, the reheating rate would be increased [21] due to the re-conversion of $\bar{\nu}_{s} \rightarrow \bar{\nu}_{e}$ and/or $\nu_{s} \rightarrow \nu_{e}$ since the $\nu_{e}$ and $\bar{\nu}_{e}$ would have higher energy compared to the standard case. Therefore, we would not get any disfavoured region but a positive effect from this conversion. However, for the discussion on the $\bar{\nu}_{e}$ signal we still would get constraints due to a different reason [37]. In this case we would not have the reduction of the $\bar{\nu}_{e}$ signal but instead we would get an increase in the expected number of events at underground detectors. This enhanced $\bar{\nu}_{e}$ signal would follow from the increase in the average energy due to the $\bar{\nu}_{s} \rightarrow \bar{\nu}_{e}$ re-conversion. This would allow us to disfavour a similar range of parameters as in Fig. 4. Finally from the $r$-process argument, we would have a different conclusion from what we obtained in Sec. III C. Roughly speaking, the allowed region $\left(Y_{e}<0.5\right)$ in Fig. 5 would now become disfavoured region whereas the disfavoured region $\left(Y_{e}>0.5\right)$ in the same plot would now become the allowed one. The reason is the following. For example, if the parameters are in the region where $Y_{e}<0.4$ in Fig. 5, only the resonant conversion at $r_{3}$ (defined in Sec. II) is adiabatic due to the fast variation at $r_{2}$ but the slower variation of the potential at $r_{3}$ (see Fig. 2). In the anti-neutrino case, the $\bar{\nu}_{s} \rightarrow \bar{\nu}_{e}$ conversion at $r_{1}$ can be neglected, as one can see from Fig. 4, for magnetic moments smaller than $10^{-15} \mu_{B}$ or so. This implies that, instead of the net effect of the $\nu_{e} \rightarrow \nu_{s}$ conversion expected in the case we considered in Sec. III.C, now we expect efficient $\nu_{s} \rightarrow \nu_{e}$ conversion, which makes the average energy of the resulting $\nu_{e}$ higher than in the no-conversion case. This has the result of driving the $Y_{e}$ value larger than 0.5 (see eq. (12)). On the other hand one can expect the opposite behaviour for the region where $Y_{e}>0.5$ (and larger magnetic moment) the conversions at all the points $r_{i}$ are expected to be adiabatic and therefore important. Thus, roughly speaking, the net effect is the reduction of $\nu_{e}$ flux, when neutrinos reach the position where the r-process is occurring. The net effect is that the $\nu_{e}$ flux is decreased and the $\bar{\nu}_{e}$ is increased. This way we expect that the value of $Y_{e}$ will become smaller than in the no-conversion case. Thus (assuming that $\nu_{s}$ and $\bar{\nu}_{s}$ are copiously produced in the core) the region with $Y_{e}>0.5$ would now become allowed by the r-process criterion.

\section{Acknowledgement}

We thank H.-T. Janka for correspondence on the magnetic field of the proto-neutron star. This work has been supported by DGICYT under Grant N. PB95-1077, by a joint CICYT-INFN grant, and by the TMR network ERBFMRXCT960090 of the European Union. H. N. has been supported by a DGICYT fellowship at Univ. de Valencia when major part of this work was done, and by a postdoctral fellowship from Fundação de Amparo à Pesquisa do Estado de São Paulo (FAPESP). R. T. has been supported by a fellowship from Generalitat Valenciana.

[1] J. T. Peltoniemi, D. Tommasini and J. W. F. Valle, Phys. Lett. B298, 383 (1993).

[2] For a recent review see J.R. Primack, astro-ph/9707285; astro-ph/9610078, E. Gawiser and J. Silk, Science, 280, 1405 (1998) astro-ph/9806197; and references therein.

[3] C. Athanassopoulos et al., Phys. Rev. Lett. 75, 2650 (1995); Phys. Rev. Lett. 77, 3082 (1996); nucl-ex/9706006.

[4] Talks given by K. Lande (for Homestake collaboration), V. Gavrin (for SAGE collaboration), T. Kirsten (for GALLEX 
collaboration) and Y. Suzuki (for SuperKamiokande collaboration), in Proceedings of the XVIII International Conference on Neutrino Physics and Astrophysics (Neutrino '98), Takayama, Japan, 4-9 June 1998, edited by Y. Suzuki and Y. Totsuka, to be published in Nucl. Phys. B (Proc. Suppl.).

[5] T. Kajita, talk at Neutrino 98 [4], ; Super-Kamiokande Collaboration, Y. Fukuda et al., Phys. Rev. Lett. 81, 1562 (1998); Phys. Lett. B433, 9 (1998); ibid. B436, 33 (1998); IMB Collaboration, R. Becker-Szendy et al., Phys. Rev. D46, 3720 (1992); Soudan Collaboration, W. W. M. Allison et al., Phys. Lett. B391, 491 (1997); H. Gallagher et al., talk given at XXIX International Conference on High Energy Physics, July 23-29, 1998, UBC, Vancouver, Canada.

[6] Q. Y. Liu and A. Yu. Smirnov, Nucl. Phys. B524, 505 (1998); V. Barger, K. Whisnant and T. Weiler, Phys.Lett. B427, 97 (1998); S. Gibbons, R. N. Mohapatra, S. Nandi and A. Raichoudhuri, Phys. Lett. B430, 296 (1998); Nucl.Phys. B524, 505 (1998); S. Bilenky, C. Giunti and W. Grimus, Eur. Phys. J. C 1, 247 (1998); S. Goswami, Phys. Rev. D 55, 2931 (1997); N. Okada and O. Yasuda, Int. J. Mod. Phys. A12, 3669 (1997).

[7] S. P. Mikheyev and A. Yu. Smirnov, Sov. Phys, JETP, 64, 4 (1986); Prog. Part. Nucl. Phys. 23, 41 (1989); K. Kainulainen, J. Maalampi and J. T. Peltoniemi, Nucl. Phys. B358, 435 (1991); J. T. Peltoniemi, Astron. \& Astrophys. 254, 121 (1992); B. Mukhopadhyaya and R. Gandhi, Phys. Rev. D46, 3682 (1992); G. Raffelt and G. Sigl, Astropart. Phys. 1, 165, (1993); X. Shi and G. Sigl, Phys. Lett. B323, 360 (1994).

[8] H. Nunokawa, J. T. Peltoniemi, A. Rossi and J. W. F. Valle, Phys. Rev. D56, 1704 (1997).

[9] J. Schechter and J.W.F. Valle, Phys. Rev. D24, 1883 (1981); ibid. D25, 283 (1982).

[10] E. Kh. Akhmedov, Phys. Lett. B213, 64 (1988); C. S. Lim and W. Marciano, Phys. Rev. D37, 1368 (1988).

[11] M. Mikheyev and A. Smirnov, Sov. J. Nucl. Phys. 42, 913 (1985); L. Wolfenstein, Phys. Rev. D20, 2634 (1979).

[12] K. Fujikawa and R. E. Shrock, Phys. Rev. Lett. 45, 963 (1980).

[13] For e.g., M. Fukugita and T. Yanagida, Phys. Rev. Lett. 58, 1807 (1987); K. S. Babu and V. S. Mathur, Phys. Lett. 196B, 218 (1987); M. B. Voloshin, Sov. J. Nucl. Phys. 48, 512 (1988); K. S. Babu and R. Mohapatra, Phys. Rev. Lett. 63, 228 (1988); S. M. Barr, E. M. Freire and A. Zee, ibid. 65, 2626 (1990); D. Chang, W.-Y.Keung, S. Lipovaca and G. Senjanovic, ibid. 67, 953 (1991).

[14] J. T. Peltoniemi and J. W. F. Valle, Nucl. Phys. B406, 409 (1993).

[15] D.O. Caldwell and R.N. Mohapatra, Phys. Rev. D48, 3259 (1993).

[16] C. Caso et al., The European Physical Journal C3, 1 (1998).

[17] I. Goldman, Y. Aharonov, G. Alexander and S. Nussinov, Phys. Rev. Lett. 60, 1789 (1988); J. M. Lattimer and J. Cooperstein, ibid. 61, 23 (1988); R. Barbieri and R. N. Mohapatra, ibid. 61, 27 (1988); D. Notzold, Phys. Rev. D38, 1658 (1988).

[18] A. Ayala, J.C. D’Olivo and M. Torres, hep-ph/9804230.

[19] K. Hirata et. al., Phys. Rev. Lett. 58, 1490 (1987).

[20] R. Bionta et. al., Phys. Rev. Lett. 58, 1494 (1987).

[21] V. B. Voloshin, Phys. Lett. 209B, 360 (1988); V. B. Voloshin, JETP Lett. 47, 501 (1988).

[22] G. G. Raffelt, Phys. Rev. Lett. 64, 2856 (1990); Astrophys. J. 365, 559 (1990); for some earlier works, see also, M. Fukugita and S. Yazaki, Phys. Rev. D36, 3817 (1987), and references therein.

[23] E.Kh. Akhmedov, invited talk given at the 4th International Solar Neutrino Conference, Heidelberg, Germany, April 1997, 
hep-ph/9705451.

[24] For a recent analysis, see M. M. Guzzo and H. Nunokawa, hep-ph/9810408.

[25] For e.g., E. Kh. Akhmedov and Z. G. Berezhiani, Nucl. Phys. B, 479 (1992); H. Athar, J. T. Peltoniemi and A. Yu. Smirnov, Phys. Rev. D51, 6647 (1995); T. Totani, K. Sato, ibid. D54, 5975 (1996); M. Burggen, ibid. D55, 5876 (1997); E. Kh. Akhmedov, A. Lanza, S. T. Petcov and D. W. Sciama, ibid. D55, 515 (1997); H. Nunokawa, Y.-Z. Qian and G. M. Fuller, ibid. D55, 3265 (1997).

[26] J. T. Peltoniemi, in ref. []]; H. Athar and J. T. Peltoniemi, Phys. Rev. D51, 5785 (1995).

[27] S. Pastor, V. B. Semikoz and J. W. F. Valle, Astropart. Phys. 3, 87, (1995); Phys. Lett. B369, 301 (1996).

[28] L. Landau, Phys. Z. Sowjetunion 246 (1932); C. Zener, Proc. R. Soc. London A137 696 (1932).

[29] W. C. Haxton, Phys. Rev. Lett. 57, 1271 (1986); S. J. Parke, Phys. Rev. Lett. 57, 1275 (1986); A. Dar et al., Phys. Rev. D35, 3607 (1987).

[30] S. A. Colgate and R. H. White, Astrophys. J. 143, 626 (1966).

[31] J. R. Wilson, Numerical Astrophysics ed. J. M. Centrella, J. M. Leblanc and R. L. Bowers (Boston, Jones and Bartlett), p.422 (1983); H. A. Bethe and J. R. Wilson, Astrophys. J. 295, 14 (1985).

[32] C. Thompson and R. C. Duncan, Astrophys. J. 408, 194 (1993).

[33] H.-T. Janka, private communication.

[34] Y.-Z. Qian et al., Phys. Rev. Lett. 71, 1965 (1993); G. Sigl, Phys. Rev. D51, 4035 (1995); Y.-Z. Qian and G. M. Fuller, Phys. Rev. D51, 1479 (1995); Phys. Rev. D52, 656 (1995); F. N. Loreti, Y.-Z. Qian, G. M. Fuller and A. B. Balantekin, Phys. Rev. D52, 6664 (1995); J. T. Peltoniemi, Proc. Third Tallinn Syposium on Neutrino Physics, Ed. I. Ots, J. Lõhmus, P. Helde and L. Palgi (Tartu, 1995) p. 103 (hep-ph/9511323); H. Nunokawa, Y.-Z. Qian, A. Rossi and J. W. F. Valle, Phys. Rev. D54, 4356 (1996); H. Nunokawa, A. Rossi and J. W. F. Valle, Nucl. Phys. B482, 481 (1996); H. Nunokawa, Y.-Z. Qian and G. M. Fuller, in ref. 25.

[35] S. E. Woosley and E. Baron, Astrophys. J. 391, 228 (1992); S. E. Woosley, Astron. Astrophys. Suppl. Ser. 97, 205 (1993); S. E. Woosley and R. D. Hoffman, Astrophys. J. 395, 202 (1992); B. S. Meyer et al., Astrophys. J. 399, 656 (1992); S. E. Woosley et al., Astrophys. J. 433, 229 (1994).

[36] Y.-Z. Qian and S. E. Woosley, Astrophys. J. 471, 331 (1996); W. Haxton, K. Langanke, Y.-Z. Qian and P. Vogel, Phys. Rev. Lett. 78, 2694 (1997); Y.-Z. Qian, W. Haxton, K. Langanke and P. Vogel, Phys. Rev. C55, 1532 (1997).

[37] A. Yu. Smirnov, D. N. Spergel and J.N.Bahcall, Phys. Rev. D49, 1389 (1994). 